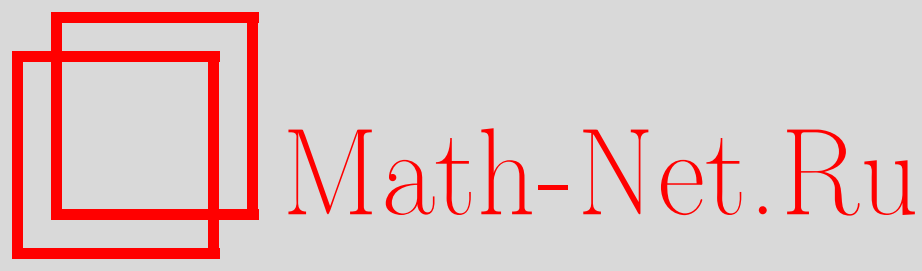

С. Дулат, Кан Ли, Цзянь-Хуа Ван, Функции Вигнера для задачи Ландау в некоммутативной квантовой механике, ТМФ, 2011, том 167, номер 2, 264-272

DOI: https://doi.org/10.4213/tmf6638

Использование Общероссийского математического портала Math-Net.Ru подразумевает, что вы прочитали и согласны с пользовательским соглашением http://www.mathnet.ru/rus/agreement

Параметры загрузки:

IP: 52.23 .180 .231

26 апреля 2023 г., 16:20:38

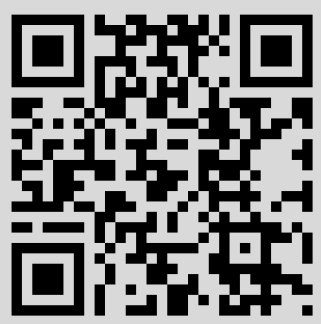




\section{ФУНКЦИИ ВИГНЕРА ДЛЯ ЗАДАЧИ ЛАНДАУ В НЕКОММУТАТИВНОЙ КВАНТОВОЙ МЕХАНИКЕ}

Исследованы функции Вигнера в некоммутативной квантовой механике. Путем решения не зависящего от времени уравнения Шредингера в некоммутативном пространстве и в некоммутативном фазовом пространстве получены функции Вигнера для задач Ландау в этих пространствах.

Ключевые слова: функция Вигнера, некоммутативная квантовая механика, задача Ландау.

\section{1. ВВЕДЕНИЕ}

В последнее время изучение физических явлений в некоммутативном пространстве (НКП) и некоммутативном фазовом пространстве (НКФП) привлекает внимание многих исследователей. Это связано с тем, что влияние пространственной некоммутативности может становиться существенным на струнном масштабе. Кроме того, имеется много работ, посвященных теоретико-полевым исследованиям различных аспектов квантовой механики в НКП и НКФП с обычными (коммутативными) временнь́ми координатами [1]-[11]. Например, фаза Ааронова-Бома в НКП и НКФП исследовалась в работах [1]-[3], а фаза Ааронова-Кашера для частиц со спином 1/2 и спином 1 в НКП и НКФП - в работах [4], [5]. В работах [6], [7] рассматривались задача Ландау и эффект Хе-Мекеллара-Вилкенса как в НКП, так и в НКФП. Имеется также ряд работ, посвященных квантовому эффекту Холла в НКП [8] и НКФП [9]. В работе [12] изучалась функция Вигнера для негамильтоновых систем в НКП. Функции Вигнера для задачи Ландау в НКП исследовались в работе [13], а функция Вигнера для осциллятора Клейна-Гордона в НКП - в работе [14]. В работе [15] рассмотрены функции Вигнера для гармонического осциллятора в случае некоммутативной квантовой механики. Функция Вигнера очень важна не только потому, что она является эквивалентом волновой функции Шредингера в квантовой механике, но и потому, что она связана с квантовым наблюдением,

\footnotetext{
*School of Physics Science and Technology, Xinjiang University, Urumqi, China

${ }^{\dagger}$ Department of Physics, Hangzhou Normal University, Hangzhou, China. E-mail: kangli60@yahoo.com.cn

${ }^{\ddagger}$ Department of Physics, Shaanxi University of Technology, Hanzhong, China
} 
и поэтому дальнейшие исследования некоммутативной функции Вигнера являются очень важными и полезными.

В настоящей работе исследуется влияние некоммутативности на функцию Вигнера для задачи Ландау. Работа построена следующим образом. В разделе 2 вводится функция Вигнера для задачи Ландау. В разделе 3 исследованы функции Вигнера для задачи Ландау в НКП. В разделе 4 с использованием обобщенного сдвига Боппа получены функции Вигнера для задачи Ландау в НКФП. Раздел 5 представляет собой заключение.

\section{2. ФУНКЦИЯ ВИГНЕРА ДЛЯ ЗАДАЧИ ЛАНДАУ}

Существуют три формулировки квантовой механики: стандартное операторное квантование, развитое Шредингером, Дираком и Гейзенбергом, квантование с помощью интеграла по траекториям, построенное Фейнманом, и формулировка в терминах фазового пространства (также известная как квантование Мойала, или деформационное квантование), развитая Вигнером [16], которая хотя и менее известна, но полезна во многих областях физики. Например, она может быть использована при описании квантового процесса переноса в фазовом пространстве, в квантовой оптике, ядерной физике, физике твердого тела, М-теории, некоммутативной геометрии и матричных моделях. В данной формулировке квантовой механики отсутствуют операторы, а наблюдаемые и амплитуды переходов являются интегралами по фазовому пространству классических числовых функций, их композиция реализуется *-произведением, и, как в статистической механике, вес задается функцией Вигнера. Исходя из квантово-механической волновой функции Вигнер построил функцию распределения, которая является вещественной, но не всюду положительной. Затем Мойал записал эволюционное уравнение для данного распределения, введя для этого свою знаменитую скобку [17]. Функция Вигнера распределения вероятности для одновременных значений координат $\mathbf{x}$ и импульсов $\mathbf{p}$ в фазовом пространстве размерности $2 d$ определяется в терминах волновой функции $\psi(\mathbf{x})$ уравнения Шредингера $\widehat{H}(\hat{\mathbf{x}}, \hat{\mathbf{p}}) \psi(\mathbf{x})=E \psi(\mathbf{x})$ (здесь $\widehat{H}$ - гамильтониан, а координаты $\hat{\mathbf{x}}$ и импульсы $\hat{\mathbf{p}}$ удовлетворяют стандартным коммутационным соотношениям $\left.\left[\hat{x}_{i}, \hat{p}_{j}\right]=i \hbar \delta_{i j}\right)$ как

$$
W(\mathbf{x}, \mathbf{p})=\frac{1}{(2 \pi)^{d}} \int_{-\infty}^{+\infty} d \mathbf{y} e^{-i \mathbf{y} \cdot \mathbf{p}} \psi^{*}\left(\mathbf{x}-\frac{\hbar}{2} \mathbf{y}\right) \psi\left(\mathbf{x}+\frac{\hbar}{2} \mathbf{y}\right),
$$

где $\psi^{*}(\mathbf{x})$ обозначает функцию, комплексно-сопряженную $\psi(\mathbf{x})$. Можно также получить не зависящую от времени функцию Вигнера чистого состояния, непосредственно решая уравнение на собственные значения в терминах *-произведения [18]:

$$
H(\mathbf{x}, \mathbf{p}) *_{\hbar} W(\mathbf{x}, \mathbf{p})=W(\mathbf{x}, \mathbf{p}) *_{\hbar} H(\mathbf{x}, \mathbf{p})=E W(\mathbf{x}, \mathbf{p}),
$$

где ассоциативое *-произведение записывается как

$$
*_{\hbar} \equiv \exp \left\{\frac{i \hbar}{2}\left(\overleftarrow{\partial}_{\mathbf{x}} \cdot \vec{\partial}_{\mathbf{p}}-\overleftarrow{\partial}_{\mathbf{p}} \cdot \vec{\partial}_{\mathbf{x}}\right)\right\}
$$

а $H(\mathbf{x}, \mathbf{p})$ - классический гамильтониан, соответствующий $\widehat{H}$. Данное произведение кодирует полное квантово-механическое действие. Если вспомнить, как действует 
трансляционный оператор, то оказывается, что *-произведение индуцирует сдвиги Боппа:

$$
\begin{aligned}
f(\mathbf{x}, \mathbf{p}) *_{\hbar} g(\mathbf{x}, \mathbf{p}) & =f\left(\mathbf{x}+\frac{i \hbar}{2} \vec{\partial}_{\mathbf{p}}, \mathbf{p}-\frac{i \hbar}{2} \vec{\partial}_{\mathbf{x}}\right) g(\mathbf{x}, \mathbf{p})= \\
& =f\left(\mathbf{x}, \mathbf{p}-\frac{i \hbar}{2} \vec{\partial}_{\mathbf{x}}\right) g\left(\mathbf{x}, \mathbf{p}+\frac{i \hbar}{2} \overleftarrow{\partial}_{\mathbf{x}}\right)
\end{aligned}
$$

Заметим, что $f(\mathbf{x}, \mathbf{p}) *_{\hbar} g(\mathbf{x}, \mathbf{p})$ обозначает квантовую деформацию обычного коммутативного произведения функций $f \cdot g$. Итак, имеются два эквивалентных способа получения функций Вигнера: с помощью равенства (1) или с помощью уравнения (2).

Для иллюстрации подхода, основанного на уравнении (2), рассмотрим задачу Ландау, задаваемую следующим гамильтонианом:

$$
\begin{aligned}
H(\mathbf{x}, \mathbf{p}) & =\frac{1}{2 \mu}\left[\left(p_{x}-\frac{e B}{2 c} y\right)^{2}+\left(p_{y}+\frac{e B}{2 c} x\right)^{2}\right]= \\
& =\frac{1}{2 \mu}\left(p_{x}^{2}+p_{y}^{2}\right)+\frac{1}{2} \mu \omega^{2}\left(x^{2}+y^{2}\right)+\omega\left(x p_{y}-y p_{x}\right),
\end{aligned}
$$

где $\omega=q B / 2 \mu c$. Переопределив координаты как

$$
q=\frac{p_{y}}{\sqrt{\mu}}+\omega \sqrt{\mu} x, \quad p=\frac{p_{x}}{\sqrt{\mu}}-\omega \sqrt{\mu} y,
$$

мы можем записать гамильтониан задачи Ландау (3) в виде, отвечающем одномерному гармоническому осциллятору, в котором $\mu=1$ и $\omega=1$ :

$$
H(q, p)=\frac{1}{2}\left(p^{2}+q^{2}\right)
$$

В терминах $q$ и $p$ задача на собственные значения (2) записывается как

$$
H(q, p) *_{\hbar} W(q, p)=W(q, p) *_{\hbar} H(q, p)=E W(q, p),
$$

где $*_{\hbar} \equiv e^{i \hbar \omega}\left(\overleftarrow{\partial}_{q} \vec{\partial}_{p}-\overleftarrow{\partial}_{p} \vec{\partial}_{q}\right)$. Отсюда

$$
\left[q^{2}+p^{2}-\hbar^{2} \omega^{2}\left(\partial_{q}^{2}+\partial_{p}^{2}\right)+2 i \hbar \omega\left(q \partial_{p}-p \partial_{q}\right)-2 E\right] W(q, p)=0
$$

Учитывая, что $W(q, p) *_{\hbar} H(q, p)=E W(q, p)$, имеем

$$
\left[q^{2}+p^{2}-\hbar^{2} \omega^{2}\left(\partial_{q}^{2}+\partial_{p}^{2}\right)-2 i \hbar \omega\left(q \partial_{p}-p \partial_{q}\right)-2 E\right] W(q, p)=0
$$

В силу соотношений (5)-(7) мнимая часть этого уравнения записывается как

$$
\left(q \partial_{p}-p \partial_{q}\right) W(q, p)=0
$$

а вещественная часть есть

$$
\left[q^{2}+p^{2}-\hbar^{2} \omega^{2}\left(\partial_{q}^{2}+\partial_{p}^{2}\right)-2 E\right] W(q, p)=0 .
$$


Вводя новую переменную

$$
\xi=\frac{q^{2}+p^{2}}{\hbar \omega}
$$

можно переписать уравнение (8) в виде

$$
\left[\frac{\xi}{4}-\xi \partial_{\xi}^{2}-\partial_{\xi}-\frac{E}{2 \hbar \omega}\right] W(\xi)=0 .
$$

Положив $W(\xi)=e^{-\xi / 2} L(\xi)$, мы переписываем уравнение (10) как

$$
\left[\xi \partial_{\xi}^{2}+(1-\xi) \partial_{\xi}+\frac{E}{2 \hbar \omega}-\frac{1}{2}\right] L(\xi)=0 .
$$

Решением этого уравнения являются полиномы Лагерра

$$
L_{n}(\xi)=\frac{1}{n !} e^{\xi} \partial_{\xi}^{n}\left(e^{-\xi} \xi^{n}\right), \quad n=\frac{E_{n}}{2 \hbar \omega}-\frac{1}{2}=0,1, \ldots, \quad E_{n}=\left(n+\frac{1}{2}\right) 2 \hbar \omega
$$

а соответствующие нормированные функции Вигнера суть

$$
W_{n}=\frac{(-1)^{n}}{\pi \hbar} e^{-\xi / 2} L_{n}(\xi)
$$

Подставляя в это равенство соотношение (9), можно записать функции Вигнера $W_{n}$ для задачи Ландау как

$$
\begin{aligned}
W_{n}(q, p) & =\frac{(-1)^{n}}{\pi \hbar} \exp \left\{-\frac{q^{2}+p^{2}}{2 \hbar \omega}\right\} L_{n}\left(\frac{q^{2}+p^{2}}{\hbar \omega}\right)= \\
& =\frac{(-1)^{n}}{\pi \hbar} \exp \left\{-\frac{H(q, p)}{\hbar \omega}\right\} L_{n}\left(\frac{2 H(q, p)}{\hbar \omega}\right) .
\end{aligned}
$$

При $n=0$ имеем

$$
W_{0}=\frac{1}{\pi \hbar} \exp \left\{-\frac{H(q, p)}{\hbar \omega}\right\} .
$$

Подставляя соотношения (4) в последние равенства, получаем функции Вигнера в переменных $\mathbf{x}, \mathbf{p}$ :

$$
\begin{aligned}
W_{n}(\mathbf{x}, \mathbf{p})= & \frac{(-1)^{n}}{\pi \hbar} \exp \left\{-\frac{\left(p_{y} / \sqrt{\mu}+\omega \sqrt{\mu} x\right)^{2}+\left(p_{x} / \sqrt{\mu}-\omega \sqrt{\mu} y\right)^{2}}{2 \hbar \omega}\right\} \times \\
& \times L_{n}\left(-\frac{\left(p_{y} / \sqrt{\mu}+\omega \sqrt{\mu} x\right)^{2}+\left(p_{x} / \sqrt{\mu}-\omega \sqrt{\mu} y\right)^{2}}{\hbar \omega}\right)= \\
= & \frac{(-1)^{n}}{\pi \hbar} \exp \left\{-\frac{H(\mathbf{x}, \mathbf{p})}{\hbar \omega}\right\} L_{n}\left(\frac{2 H(\mathbf{x}, \mathbf{p})}{\hbar \omega}\right), \\
W_{0}(\mathbf{x}, \mathbf{p})= & \frac{1}{\pi \hbar} \exp \left\{-\frac{H(\mathbf{x}, \mathbf{p})}{\hbar \omega}\right\} .
\end{aligned}
$$




\section{3. ФУНКЦИЯ ВИГНЕРА ДЛЯ ЗАДАЧИ ЛАНДАУ В НКП}

Операторы координаты $\hat{x}_{i}^{\text {nc }}$ и импульса $\hat{p}_{i}^{\text {nc }}, i=x, y$, на некоммутативной плоскости удовлетворяют коммутационным соотношениям

$$
\left[\hat{x}_{i}^{\mathrm{nc}}, \hat{x}_{j}^{\mathrm{nc}}\right]=i \theta_{i j}, \quad\left[\hat{p}_{i}, \hat{p}_{j}\right]=0, \quad\left[\hat{x}_{i}^{\mathrm{nc}}, \hat{p}_{j}\right]=i \hbar \delta_{i j} .
$$

Уравнение Шредингера в НКП имеет вид

$$
\widehat{H}(\hat{\mathbf{x}}, \hat{\mathbf{p}}) *_{\theta} \psi^{\mathrm{nc}}(\mathbf{x})=E \psi^{\mathrm{nc}}(\mathbf{x}),
$$

где произведение Мойала-Вейля (или *-произведение) определяется следующим образом: $*_{\theta}=e^{(i \theta / 2)\left(\overleftarrow{\partial}_{x} \vec{\partial}_{y}-\overleftarrow{\partial}_{y} \vec{\partial}_{x}\right)}$. После того как из уравнения $(13)$ найдена $\psi^{\mathrm{nc}}(\mathbf{x})$, функция Вигнера на НКП принимает вид

$$
W^{\mathrm{nc}}(\mathbf{x}, \mathbf{p})=\frac{1}{(2 \pi)^{2}} \int d \mathbf{y} e^{-i \mathbf{y} \cdot \mathbf{p}} \psi^{\mathrm{nc}}\left(\mathbf{x}+\frac{\hbar}{2} \mathbf{y}\right) *_{\theta} \psi^{* \mathrm{nc}}\left(\mathbf{x}-\frac{\hbar}{2} \mathbf{y}\right) .
$$

Функцию Вигнера на НКП можно также получить альтернативным способом, решая следующее уравнение на собственные значения в терминах *-произведения:

$$
H(\mathbf{x}, \mathbf{p}) * W^{\mathrm{nc}}(\mathbf{x}, \mathbf{p})=W^{\mathrm{nc}}(\mathbf{x}, \mathbf{p}) * H(\mathbf{x}, \mathbf{p})=E W^{\mathrm{nc}}(\mathbf{x}, \mathbf{p}),
$$

где

$$
*=*_{\hbar * \theta}=\exp \left(\frac{i \hbar}{2} \sum_{i=x}^{y}\left(\overleftarrow{\partial}_{x_{i}} \vec{\partial}_{p_{i}}-\overleftarrow{\partial}_{p_{i}} \vec{\partial}_{x_{i}}\right)+\frac{i \theta}{2}\left(\overleftarrow{\partial}_{x} \vec{\partial}_{y}-\overleftarrow{\partial}_{y} \vec{\partial}_{x}\right)\right)
$$

Вместо решения уравнения Шредингера в НКП с использованием *-произведения мы используем метод сдвига Боппа, т. е. заменяем $*_{\theta}$-произведение в уравнении Шредингера обычным произведением, производя при этом сдвиг

$$
\hat{x}_{i}^{\mathrm{nc}}=\hat{x}_{i}-\frac{1}{2 \hbar} \theta_{i j} \hat{p}_{j}, \quad \hat{p}_{i}^{\mathrm{nc}}=\hat{p}_{i}, \quad i=x, y, \quad \theta_{i j}=\theta \epsilon_{i j} .
$$

Тогда уравнение (13) принимает вид $\widehat{H}\left(\hat{\mathbf{x}}^{\mathrm{nc}}, \hat{\mathbf{p}}^{\mathrm{nc}}\right) \psi^{\mathrm{nc}}(\mathbf{x})=E \psi^{\mathrm{nc}}(\mathbf{x})$. Поэтому уравнение (14) можно переписать как

$$
H\left(\mathbf{x}^{\mathrm{nc}}, \mathbf{p}^{\mathrm{nc}}\right) *_{\hbar} W^{\mathrm{nc}}(\mathbf{x}, \mathbf{p})=W^{\mathrm{nc}}(\mathbf{x}, \mathbf{p}) *_{\hbar} H\left(\mathbf{x}^{\mathrm{nc}}, \mathbf{p}^{\mathrm{nc}}\right)=E W^{\mathrm{nc}}(\mathbf{x}, \mathbf{p}),
$$

где

$$
\begin{aligned}
H\left(\mathbf{x}^{\mathrm{nc}}, \mathbf{p}^{\mathrm{nc}}\right) & =\frac{1}{2 \mu}\left[\left(p_{x}-\frac{e B}{2 c} y^{\mathrm{nc}}\right)^{2}+\left(p_{y}+\frac{e B}{2 c} x^{\mathrm{nc}}\right)^{2}\right]= \\
& =\frac{1}{2 \mu}\left\{\left[\left(1-\frac{e B}{4 \hbar c} \theta\right) p_{x}-\frac{e B}{2 c} y\right]^{2}+\left[\left(1-\frac{e B}{4 \hbar c} \theta\right) p_{y}+\frac{e B}{2 c} x\right]^{2}\right\}= \\
& =\frac{1}{2 \mu}\left\{\left[(1+\kappa) p_{x}-\omega y\right]^{2}+\left[(1+\kappa) p_{y}+\omega x\right]^{2}\right\}, \quad \kappa=-\frac{q B}{4 \hbar c} \theta .
\end{aligned}
$$

Переопределяя координаты,

$$
q^{\mathrm{nc}}=\frac{(1+\kappa) p_{y}}{\sqrt{\mu}}+\omega \sqrt{\mu} x, \quad p^{\mathrm{nc}}=\frac{(1+\kappa) p_{x}}{\sqrt{\mu}}-\omega \sqrt{\mu} y,
$$


можно записать гамильтониан (17) для задачи Ландау в виде, отвечающем одномерному гармоническому осциллятору, в котором $\mu=1$ и $\omega=1$ :

$$
H\left(q^{\mathrm{nc}}, p^{\mathrm{nc}}\right)=\frac{1}{2}\left(\left(p^{\mathrm{nc}}\right)^{2}+\left(q^{\mathrm{nc}}\right)^{2}\right) .
$$

Сравнивая уравнения (2) и (16), получаем

$$
W_{n}^{\mathrm{nc}}(q, p)=\left.W(q, p)\right|_{\substack{q=q^{\mathrm{nc}} \\ p=p^{\mathrm{nc}}}},
$$

откуда

$$
\begin{aligned}
W_{n}^{\mathrm{nc}}\left(q^{\mathrm{nc}}, p^{\mathrm{nc}}\right) & =\frac{(-1)^{n}}{(\pi \hbar)^{2}} \exp \left\{-\frac{\left(q^{\mathrm{nc}}\right)^{2}+\left(p^{\mathrm{nc}}\right)^{2}}{2 \hbar \omega(1+\kappa)}\right\} L_{n}\left(\frac{\left(q^{\mathrm{nc}}\right)^{2}+\left(p^{\mathrm{nc}}\right)^{2}}{\hbar \omega(1+\kappa)}\right)= \\
& =\frac{(-1)^{n}}{(\pi \hbar)^{2}} \exp \left\{-\frac{H\left(q^{\mathrm{nc}}, p^{\mathrm{nc}}\right)}{\hbar \omega(1+\kappa)}\right\} L_{n}\left(\frac{2 H\left(q^{\mathrm{nc}}, p^{\mathrm{nc}}\right)}{\hbar \omega(1+\kappa)}\right) .
\end{aligned}
$$

При $n=0$ имеем

$$
W_{0}^{\mathrm{nc}}=\frac{1}{(\pi \hbar)^{2}} \exp \left\{-\frac{H\left(q^{\mathrm{nc}}, p^{\mathrm{nc}}\right)}{\hbar \omega(1+\kappa)}\right\} .
$$

\section{4. ФУНКЦИЯ ВИГНЕРА ДЛЯ ЗАДАЧИ ЛАНДАУ В НКФП}

Случай, когда и операторы координаты, и операторы импульса не коммутируют между собой [10], [11], отличается от случая, когда только операторы координаты не коммутируют между собой. Таким образом, в НКФП не только операторы координаты являются некоммутирущими, как в (12), но и операторы импульса удовлетворяют коммутационным соотношениям $\left[\hat{p}_{i}^{\text {ncps }}, \hat{p}_{j}^{\text {ncps }}\right]=i \bar{\theta}_{i j}, i, j=x, y$. Здесь $\left\{\bar{\theta}_{i j}\right\}-$ полностью антисимметричная матрица, определяющая свойство некоммутативности импульсов в НКФП и играющая роль, аналогичную постоянной $\hbar$ в обычной квантовой механике. Уравнение Шредингера в НКФП записывается в виде

$$
\widehat{H}(\hat{\mathbf{x}}, \hat{\mathbf{p}}) *_{\theta} *_{\bar{\theta}} \psi^{\mathrm{ncps}}(\mathbf{x})=E \psi^{\mathrm{ncps}}(\mathbf{x}),
$$

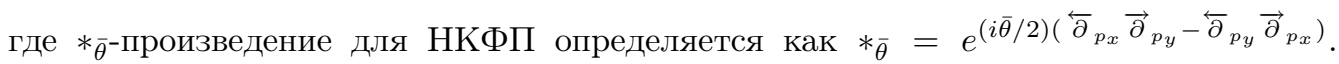
Функция Вигнера на НКФП записывается в виде

$$
W^{\mathrm{ncps}}(\mathbf{x}, \mathbf{p})=\frac{1}{(2 \pi)^{2}} \int d \mathbf{y} e^{-i \mathbf{y} \cdot \mathbf{p}} \psi^{\mathrm{ncps}}\left(\mathbf{x}+\frac{\hbar}{2} \mathbf{y}\right) *_{\theta} *_{\bar{\theta}} \psi^{* \mathrm{ncps}}\left(\mathbf{x}-\frac{\hbar}{2} \mathbf{y}\right),
$$

а уравнение на $*$-собственные значения в НКФП таково:

$$
H(\mathbf{x}, \mathbf{p}) * W(\mathbf{x}, \mathbf{p})=W(\mathbf{x}, \mathbf{p}) * H(\mathbf{x}, \mathbf{p})=E W(\mathbf{x}, \mathbf{p}),
$$

где $*=*_{\hbar} *_{\theta} *_{\bar{\theta}}$.

Вместо решения уравнения Шредингера в НКФП мы используем метод сдвига Боппа, т. е. заменяем $*_{\theta} *_{\bar{\theta}}$-произведение в уравнении Шредингера обычным произведением, сделав сдвиг

$$
\hat{x}_{i}^{\mathrm{ncps}}=\alpha \hat{x}_{i}-\frac{1}{2 \alpha \hbar} \theta_{i j} \hat{p}_{j}, \quad p_{i}^{\mathrm{ncps}}=\alpha \hat{p}_{i}+\frac{1}{2 \alpha \hbar} \bar{\theta}_{i j} \hat{x}_{j}, \quad i=x, y,
$$


где $\bar{\theta}_{i j}=\bar{\theta} \epsilon_{i j}, \theta \bar{\theta}=4 \hbar^{2} \alpha^{2}\left(1-\alpha^{2}\right)$. Тогда уравнение (19) принимает вид

$$
\widehat{H}\left(\hat{\mathbf{x}}^{\mathrm{ncps}}, \hat{\mathbf{p}}^{\mathrm{ncps}}\right) \psi^{\mathrm{ncps}}(\mathbf{x})=E \psi^{\mathrm{ncps}}(\mathbf{x}),
$$

а уравнение (20) можно переписать как

$$
H\left(\mathbf{x}^{\mathrm{ncps}}, \mathbf{p}^{\mathrm{ncps}}\right) *_{\hbar} W^{\mathrm{ncps}}(\mathbf{x}, \mathbf{p})=W^{\mathrm{ncps}}(\mathbf{x}, \mathbf{p}) *_{\hbar} H\left(\mathbf{x}^{\mathrm{ncps}}, \mathbf{p}^{\mathrm{ncps}}\right)=E W^{\mathrm{ncps}}(\mathbf{x}, \mathbf{p}) .
$$

Гамильтониан для двумерной задачи Ландау в НКФП в симметричной калибровке имеет вид

$$
\begin{aligned}
H\left(\mathbf{x}^{\mathrm{ncps}}, \mathbf{p}^{\mathrm{ncps}}\right)= & \frac{1}{2 \mu}\left[\left(p_{x}^{\mathrm{ncps}}-\frac{e B}{2 c} y^{\mathrm{ncps}}\right)^{2}+\left(p_{y}^{\mathrm{ncps}}+\frac{e B}{2 c} x^{\mathrm{ncps}}\right)^{2}\right]= \\
= & \frac{1}{2 \mu}\left\{\left[\left(\alpha+\frac{\kappa}{\alpha}\right) p_{x}-\omega\left(\alpha+\frac{\lambda}{\alpha}\right) y\right]^{2}+\right. \\
& \left.+\left[\left(\alpha+\frac{\kappa}{\alpha}\right) p_{y}+\omega\left(\alpha+\frac{\lambda}{\alpha}\right) x\right]^{2}\right\}
\end{aligned}
$$

где $\lambda=-\bar{\theta} / 2 \hbar \omega$. Переопределяя координаты,

$$
\begin{aligned}
& q^{\mathrm{ncps}}=\frac{(\alpha+\kappa / \alpha) p_{y}}{\sqrt{\mu}}+\omega\left(\alpha+\frac{\lambda}{\alpha}\right) \sqrt{\mu} x, \\
& p^{\mathrm{ncps}}=\frac{(\alpha+\kappa / \alpha) p_{x}}{\sqrt{\mu}}-\omega\left(\alpha+\frac{\lambda}{\alpha}\right) \sqrt{\mu} y,
\end{aligned}
$$

можно записать гамильтониан (19) задачи Ландау в НКФП в виде, отвечающем одномерному гармоническому осциллятору, в котором $\mu=1$ и $\omega=1$ :

$$
H\left(q^{\mathrm{ncps}}, p^{\mathrm{ncps}}\right)=\frac{1}{2}\left(\left(p^{\mathrm{ncps}}\right)^{2}+\left(q^{\mathrm{ncps}}\right)^{2}\right) .
$$

Сравнивая уравнения (2) и (22), получаем

$$
W_{n}^{\text {ncps }}(q, p)=\left.W(q, p)\right|_{\substack{q=q^{\text {ncps }} \\ p=p^{\text {ncps }}}}
$$

откуда

$$
\begin{aligned}
W_{n}^{\mathrm{ncps}}(q, p)= & \frac{(-1)^{n}}{(\pi \hbar)^{2}} \exp \left\{-\frac{\left(q^{\mathrm{ncps}}\right)^{2}+\left(p^{\mathrm{ncps}}\right)^{2}}{2 \hbar \omega(\alpha+\kappa / \alpha)(\alpha+\lambda / \alpha)}\right\} \times \\
& \times L_{n}\left(\frac{\left(q^{\mathrm{ncps}}\right)^{2}+\left(p^{\mathrm{ncps}}\right)^{2}}{\hbar \omega(\alpha+\kappa / \alpha)(\alpha+\lambda / \alpha)}\right)= \\
= & \frac{(-1)^{n}}{(\pi \hbar)^{2}} \exp \left\{-\frac{H\left(q^{\mathrm{nc}}, p^{\mathrm{nc}}\right)}{\hbar \omega(\alpha+\kappa / \alpha)(\alpha+\lambda / \alpha)}\right\} \times \\
& \times L_{n}\left(\frac{2 H\left(q^{\mathrm{ncps}}, p^{\mathrm{ncps}}\right)}{\hbar \omega(\alpha+\kappa / \alpha)(\alpha+\lambda / \alpha)}\right)
\end{aligned}
$$


- функция Вигнера для задачи Ландау в НКФП. Функция Вигнера, соответствующая волновой функции основного состояния, имеет вид

$$
W_{0}^{\mathrm{ncps}}=\frac{1}{(\pi \hbar)^{2}} \exp \left\{-\frac{H\left(q^{\mathrm{ncps}}, p^{\mathrm{ncps}}\right)}{\hbar \omega(\alpha+\kappa / \alpha)(\alpha+\lambda / \alpha)}\right\} .
$$

Следует упомянуть, что преобразование (24), а также общий вид (25) функции Вигнера задачи Ландау в НКФП представляют собой полностью новые результаты.

\section{5. ЗАКЛЮЧИТЕЛЬНЫЕ ЗАМЕЧАНИЯ}

Мы исследовали функции Вигнера для задачи Ландау в обычном коммутативном пространстве, а затем для случаев НКП и НКПФ. Вместо утомительных вычислений с использованием *-произведения мы использовали метод "сдвига", пользуясь тем, что *-произведение в уравнениях (13) и (19) можно заменить уравнениями сдвига Боппа (15) в НКП и (21) в НКФП. Эти сдвиги эквивалентны использованию *-произведения. Дополнительные члены в функции Вигнера (18) на НКП и в функции Вигнера (25) на НКФП связаны с некоммутативностью пространства и фазового пространства. Ожидается, что этот эффект можно будет проверить при очень высоких энергиях, так что его экспериментальное наблюдение остается предметом дальнейших исследований.

Использованный в работе метод можно применить к другим физическим задачам в НКП и НКФП. Этому будут посвящены наши дальнейшие работы.

Благодарности. Мы благодарим профессора Ранджбар-Даеми за любезное приглашение и гостеприимство во время визита авторов в International Centre for Theoretical Physics (Triest, Italy). Настоящая работа выполнена при поддержке National Natural Science Foundation of China (проекты № 10965006, 10875035) и Natural Science Foundation of Zhejiang Provence (проект Y6110470).

\section{Список литературы}

[1] M. Chaichian, M. M. Sheikh-Jabbari, A. Tureanu, Phys. Rev. Lett., 86:13 (2001), 2716-2719, arXiv: hep-th/0010175; M. Chaichian, A. Demichev, P. Prešnajder, M. M. Sheikh-Jabbari, A. Tureanu, Nucl. Phys. B, 611:1-3 (2001), 383-402, arXiv: hep-th/0101209.

[2] M. Chaichian, P. Prešnajder, M. M. Sheikh-Jabbari, A. Tureanu, Phys. Lett. B, 527:1-2 (2002), 149-154, arXiv: hep-th/0012175; H. Falomir, J. Gamboa, M. Loewe, F. Mendez, J. C. Rojas, Phys. Rev. D, 66:4 (2002), 045018, 13 pp., arXiv: hep-th/0203260.

[3] K. Li, S. Dulat, Eur. Phys. J. C, 46:3 (2006), 825-828, arXiv: hep-th/0508193.

[4] B. Mirza, M. Zarei, Eur. Phys. J. C, 32:4 (2004), 583-586, arXiv: hep-th/0311222; K. Li, J. Wang, Eur. Phys. J. C, 50:4 (2007), 1007-1011, arXiv: hep-th/0608100.

[5] B. Mirza, R. Narimani, M. Zarei, Eur. Phys. J. C, 48:2 (2006), 641-645, arXiv: hep-th/0610323; S. Dulat, K. Li, Eur. Phys. J. C, 54:2 (2008), 333-337, arXiv: 0802.1243.

[6] J. Gamboa, F. Méndez, M. Loewe, J. C. Rojas, Modern Phys. Lett. A, 16:32 (2001), 2075-2078, arXiv: hep-th/0104224; P. A. Horváthy, Ann. Phys., 299:1 (2002), 128-140, arXiv: hep-th/0201007; Ö. F. Dayi, L. T. Kelleyane, Modern Phys. Lett. A, 17:29 (2002), 1937-1944, arXiv: hep-th/0202062; S. Dulat, K. Li, Chin. Phys. C, 32:2 (2008), 92-95; P. R. Giri, P. Roy, Eur. Phys. J. C, 57:4 (2008), 835-839, arXiv: 0803.4090. 
[7] J. Wang, K. Li, J. Phys. A, 40:9 (2007), 2197-2202, arXiv:0708.0783; S. Dulat, K. Li, J. Wang, J. Phys. A, 41:6 (2008), 065303, 7 pp., arXiv:0802.1116; Chin. Phys. B, 17:5 (2008), 1716-1719.

[8] Ö.F. Dayi, A. Jellal, J. Math. Phys., 43:10 (2002), 4592-4601, arXiv: hep-th/0111267; Erratum 45:2 (2004), 827; A. Kokado, T. Okamura, T. Saito, Hall effect in Noncommutative spaces, arXiv: hep-th/0307120; B. Basu, S. Ghosh, Phys. Lett. A, 346:1-3 (2005), 133-140, arXiv: cond-mat/0503266; B. Harms, O. Micu, J. Phys. A, 40:33 (2007), 10337-10347, arXiv: hep-th/0610081; A. L. Carey, K. Hannabuss, V. Mathai, J. Geom. Symmetry Phys., 6 (2006), 16-37, arXiv: math/0008115.

[9] S. Dulat, K. Li, Eur. Phys. J. C, 60:1 (2009), 163-168, arXiv: 0802.1118.

[10] M. Demetrian, D. Kochan, Acta Phys. Slovaca, 52:1 (2002), 1-9, arXiv: hep-th/0102050; Z. Jian-Zu, Phys. Lett. B, 584:1-2 (2004), 204-209, arXiv: hep-th/0405135.

[11] K. Li, J. Wang, C. Chen, Modern Phys. Lett. A, 20:28 (2005), 2165-2174, arXiv: hep-th/0409234.

[12] T. Heng, B. Lin, S. Jing, Chin. Phys. Lett., 27:9 (2010), 090302, 4 pp.

[13] Ö.F. Dayi, L. T. Kelleyane, Modern Phys. Lett. A, 17:29 (2002), 1937-1944, arXiv: hep-th/0202062.

[14] K. Li, J. Wang, S. Dulat, K. Ma, Internat. J. Theoret. Phys., 49:1 (2010), 134-143.

[15] J. Wang, K. Li, S. Dulat, Wigner Functions for harmonic oscillator in noncommutative phase space, arXiv: 0908.1703.

[16] E. Wigner, Phys. Rev., 40:50 (1932), 749-759.

[17] J. E. Moyal, Proc. Camb. Philos. Soc., 45:1 (1949), 99-124.

[18] D. B. Fairlie, C. A. Manogue, J. Phys. A, 24:16 (1991), 3807-3815; T. Curtright, D. Fairlie, C. Zachos, Phys. Rev. D, 58:2 (1998), 025002, 14 pp., arXiv: hep-th/9711183; C. Zachos, Internat. J. Modern Phys. A, 17:3 (2002), 297-316, arXiv: hep-th/0110114.

Поступила в редакцию 26.08.2010, после доработки 27.10.2010 